\title{
Small bowel gastrointestinal stromal tumor: a retrospective study of 32 cases at a single center and review of the literature
}

This article was published in the following Dove Press journal:

Therapeutics and Clinical Risk Management

\begin{abstract}
Lei Zhou',*
Yusheng Liaol,*

jie $\mathrm{Wu}^{1,2}$

Jing Yang'

Heng Zhang'

Xiangyang Wang ${ }^{3}$

Shengbin Sun ${ }^{1,4}$

'Department of Gastroenterology, The Central Hospital of Wuhan,

Tongji Medical College, Huazhong University of Science and Technology,

Wuhan, Hubei, Peoples' Republic of

China; ${ }^{2}$ Key Laboratory for Molecular

Diagnosis of Hubei Province,

The Central Hospital of Wuhan,

Tongji Medical College, Huazhong

University of Science and Technology,

Wuhan, Peoples' Republic of China;

${ }^{3}$ Department of Gastrointestinal

Surgery, The Central Hospital of

Wuhan, Tongji Medical College,

Huazhong University of Science and

Technology, Wuhan, Hubei, Peoples'

Republic of China; ${ }^{4}$ Clinical Research

Center of Digestive Endoscopy, The

Central Hospital of Wuhan, Tongji

Medical College, Huazhong University

of Science and Technology, Wuhan,

Hubei, Peoples' Republic of China

*These authors contributed equally to this work
\end{abstract}

Correspondence: Shengbin Sun

Department of Gastroenterology and Clinical Research Center of Digestive Endoscopy, The Central Hospital of Wuhan, Tongji Medical College, Huazhong University of Science and Technology, 26 Shengli Street, Jiangan

District, Wuhan, Hubei 4300I4,

Peoples' Republic of China

Tel +8627822 II 66

Fax +86 27881877 19

Email shengbinsun2006@sina.com
Background: Gastrointestinal stromal tumor (GIST) is a rare tumor of the small bowel, which can be difficult to diagnose and has a varied clinical outcome.

Purpose: This is a retrospective review of the diagnosis, management, and clinical outcome of 32 patients diagnosed with primary small bowel GIST from a single center and a comparison of the findings with previously published cases.

Patients and methods: Retrospective review of data from patient clinical records, endoscopic and imaging findings, surgical procedures, tumor histology and immunohistochemistry, and clinical outcome was conducted.

Results: Data of 32 patients with a median age of 56 years including $50 \%$ men and women were reviewed. The majority (29/32) were symptomatic at presentation, with the main symptom being gastrointestinal bleeding (15/32). Imaging detection rates included ultrasound $(0 \%)$, magnetic resonance imaging $(0 \%)$, computed tomography $(54.8 \%)$, computed tomography angiography $(71.4 \%)$, and double-balloon enteroscopy $(88.9 \%)$. The mean tumor diameter was $5.3 \mathrm{~cm}$; 4 tumors were located in the duodenum, 21 in the jejunum, and 7 in the ileum. Based on the tumor size and mitotic index, 5 (15.6\%), 15 (46.9\%), $0(0 \%)$, and $12(37.5 \%)$ patients were classified into very low-risk, low-risk, intermediate-risk, and high-risk groups. Immunohistochemistry showed positive expression for CD1 17 (100\%), CD34 (81.2\%), DOG1 (93.8\%), smooth muscle actin (37.5\%), S100 (9.4\%), and desmin (6.2\%). Twenty-five patients (78.1\%) were treated with open surgical tumor resection; seven patients $(21.9 \%)$ underwent laparoscopic surgery. Postoperative complications that occurred in seven patients (21.9\%) were resolved with conservative management. Four patients were treated with postoperative imatinib. At median follow-up of 30 months, two patients were died.

Conclusion: The findings from this case series, combined with the findings from previously published cases, provide an update on the current status of the diagnosis and the therapeutic approaches that might lead to improvement in prognosis for patients who present with primary small bowel GIST.

Keywords: small bowel, gastrointestinal stromal tumor, diagnosis, prognosis, clinical outcome

\section{Introduction}

Although rare, gastrointestinal stromal tumor (GIST) is the most common primary mesenchymal tumor of the gastrointestinal (GI) system and is derived from cells showing morphological and immunophenotypic similarities with the interstitial cells of Cajal. ${ }^{1}$ The annual incidence of GIST is between 11 and 14.5 cases per million, and GIST accounts for between 1\% and 3\% of all GI neoplasms. ${ }^{2,3}$ Primary GIST arising in the GI tract presents symptomatically in $69 \%$ of cases, and is diagnosed incidentally 
at surgery in $21 \%$ of cases, with the remaining $10 \%$ found incidentally at autopsy. ${ }^{3}$

CD117, the c-kit proto-oncogene protein, is a tyrosine kinase growth factor receptor and is the most specific and important immunohistochemical tissue marker for GIST. ${ }^{4}$ The use of histopathology and immunohistochemical staining together with other immunomarkers, including CD34, DOG1, smooth muscle actin (SMA), S100 protein, and desmin, have helped to distinguish GIST from other primary mesenchymal tumors of the GI tract. ${ }^{4}$

Although GIST can arise in any portion of the GI tract, from the esophagus to the rectum, the small bowel is the second most common site of involvement (30\%-40\%), after the stomach $(40 \%-60 \%) .{ }^{5}$ Small bowel GIST is one of the most common tumors of the small bowel. ${ }^{6,7}$ The diagnosis of GIST of the small bowel may be delayed for several reasons, including its relatively low incidence, nonspecific and variable symptoms, the wide spectrum of radiological appearances, intestinal thickening, and the presence of overlapping loops of intestine, which make imaging studies difficult; all of these lead to delayed or misdiagnosis of GIST of the small bowel. ${ }^{8-10}$

The aim of this retrospective case study was to review the diagnosis, management, and clinical outcome of 32 patients diagnosed with primary small bowel GIST from a single center in Wuhan, China. The findings, supported by a literature review of previous cases, aimed to contribute to knowledge of the therapeutic approach and improvement of prognosis in patients who present with primary small bowel GIST.

\section{Patients and methods}

\section{Ethics approval and patient consent}

Written informed consent was obtained from all patients included in the study. The study was approved by the Ethics and Research Committee of The Central Hospital of Wuhan, China.

\section{Patient selection}

The medical records of patients diagnosed with primary GIST of the small bowel who were admitted to the Central Hospital of Wuhan between January 2013 and December 2017 were retrospectively reviewed. The following inclusion criteria were used for patients in this study: the tumors were located in the small bowel, which were confirmed by preoperative imaging or endoscopy or a surgical procedure; all patients underwent surgical tumor excision; and patients had histologically confirmed small bowel GIST confirmed by routine light microscopy and immunohistochemistry.
The exclusion criteria were as follows: patients with unresected tumors due to the presence of comorbid conditions preventing surgery or due to metastases, low income or lack of social support, and patients with small bowel GIST who had other coexistent malignancies. Thirty-two patients were included and analyzed in this study.

\section{Imaging studies and endoscopic techniques}

Imaging procedures used to detect small bowel GIST for some, but not all, patients included ultrasound, computed tomography (CT), CT angiography (CTA), and magnetic resonance imaging (MRI). Four commonly used endoscopic diagnostic procedures to detect small bowel GIST for some, but not all, patients included esophagogastroduodenoscopy (EGD), colonoscopy, capsule endoscopy (CE) and doubleballoon endoscopy (DBE).

\section{Data collection}

Detailed clinical and histopathologic information was collected based on case records, operation notes, and pathology reports. The clinical variables included patient demographics, symptoms on admission, the diagnostic methods used, and their findings (including tumor location [duodenum, jejunum, or ileum], tumor size, tumor outline, tumor margin, and tumor growth pattern [exophytic, intraluminal, or combined]). The surgical procedures used and their findings were included.

\section{Histopathology and immunohistochemistry}

Routine diagnostic histopathology was performed on the tumor resection specimens. The histological features recorded included tumor necrosis, tumor surface erosion or ulceration, and tumor cell type (spindle cell type, epithelioid cell type, and mixed spindle-epithelioid type). The mitotic index was identified as the number of mitotic figures per 50 high-power fields (HPFs) by light microscopy.

The routine diagnostic immunohistochemistry technique used a panel of seven primary antibodies purchased from Boster Biological Technology (Wuhan, China), which included a mouse anti-CD117 monoclonal antibody used at 1:100 dilution, a rabbit anti-CD34 polyclonal antibody used at 1:200 dilution, a mouse anti-DOG1 monoclonal antibody used at 1:200 dilution, a mouse anti-SMA monoclonal antibody used at 1:150 dilution, a mouse anti-S100 monoclonal antibody used at 1:50 dilution, a mouse anti-desmin monoclonal antibody used at 1:100 dilution, and a rabbit anti-Ki-67 monoclonal antibody used at 1:100 dilution. 
Based on tumor size and mitotic index, tumor risk categories were evaluated according to the modified National Institutes of Health (NIH) classification system proposed by Joensuu. ${ }^{11}$ The NIH tumor risk categories used were very low-risk, low-risk, intermediate-risk, and high-risk groups.

\section{Patient follow-up}

Follow-up results were obtained by telephone calls with the patients or their close relatives, and outpatient clinic visits after the patients were discharged from the hospital. Intermediate- and high-risk patients underwent abdominal CT scan or MRI every 3 or 4 months for the first 3 years after surgery, then every 6 months until the fifth year. Very low- and low-risk patients underwent CT or MRI examination every 6-12 months for 5 years after surgery. The last follow-up evaluation for patients in the study was on December 31, 2017.

\section{Statistical analysis}

Data were presented as the median, mean, percentage, and the number of cases. All statistical analysis was performed using SPSS version 17.0 (SPSS Inc., Chicago, IL, USA).

\section{Results \\ Clinical characteristics of 32 patients diagnosed with primary GIST of the small bowel}

From January 2013 to December 2017, 32 patients met the inclusion criteria for this retrospective study. The general characteristics of these patients are summarized in Table 1. The median age at presentation was 56 years (range, $23-81$ years). There were 22 patients $(68.7 \%)$ who were younger than

Table I General characteristics of the 32 patients studied

\begin{tabular}{ll}
\hline Characteristics & Results \\
\hline Gender & \\
Male & $16(50 \%)$ \\
Female & $16(50 \%)$ \\
Age (years) & \\
$<50$ & $8(25 \%)$ \\
50 to $<60$ & $14(43.7 \%)$ \\
60 to $<70$ & $6(18.8 \%)$ \\
70 to $<80$ & $3(9.4 \%)$ \\
$>80$ & $1(3.1 \%)$ \\
Age range (years) & $23-81$ \\
Median age (years) & 56 \\
Symptoms & \\
Gastrointestinal bleeding & $15(46.8 \%)$ \\
Abdominal pain & $6(18.8 \%)$ \\
Abdominal mass & $4(12.5 \%)$ \\
Abdominal distension & $3(9.4 \%)$ \\
Anemia & $1(3.1 \%)$ \\
Incidental finding & $3(9.4 \%)$ \\
\hline
\end{tabular}

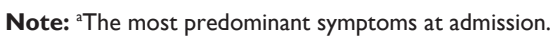

60 years; 16 patients $(50 \%)$ were male, and 16 patients $(50 \%)$ were female. There were 29 patients $(90.6 \%)$ who were symptomatic at presentation, with the most common symptom being GI bleeding in 15 patients $(46.8 \%)$, followed by abdominal pain in 6 patients $(18.8 \%)$. The other presenting symptoms were an abdominal mass in four patients (12.5\%), abdominal distension in three patients $(9.4 \%)$, and anemia in one patient $(3.1 \%)$. Three tumors $(9.4 \%)$ were detected incidentally during medical imaging for other reasons (Figures 2A and 7A).

\section{Clinical imaging and surgical findings in 32 patients diagnosed with primary GIST of the small bowel}

Ultrasonography examination was performed in nine patients $(28.1 \%)$ who presented with abdominal mass or pain. Eight abdominal or pelvic space-occupying lesions were detected by ultrasound, but three of these cases were misinterpreted, using ultrasound, as gynecological tumors, and five masses could not be identified by ultrasound as to their location or possible diagnosis (Figure 1A1 and C1). In one patient with small bowel obstruction and ascites, ultrasound was not able to identify the lesion.

CT scan was the most commonly performed imaging tool in this study, being performed for 31 patients (96.9\%). CT imaging provided a provisional diagnosis of GIST of the small bowel in 17 patients (54.8\%) out of the 32 cases included in this study (Figures 1C2, C3, 4A1, A2, and $5 \mathrm{C} 1, \mathrm{C} 2$ ). Five tumors were missed in $\mathrm{CT}$ (Figures $3 \mathrm{~A} 1$ and $5 \mathrm{~A} 1, \mathrm{~B} 1$ ), five tumors did not have a confirmed site of origin (Figure 2A1 and A2), and four tumors were misinterpreted on imaging as hemangioma (Figure 7A), pancreatic tumor (Figure 2C1-C3), or as possible gynecological tumors.

CTA was performed in seven patients $(21.9 \%)$ who presented with melena $(n=5)$, abdominal pain $(n=1)$, and distension $(\mathrm{n}=1)$. CTA imaging provided a provisional diagnosis of GIST of the small bowel in five patients $(71.4 \%$; Figures $3 \mathrm{C}$ and $5 \mathrm{C} 3, \mathrm{C} 4)$. CTA demonstrated two jejunal lesions in patients who presented with abdominal pain and melena, but they were preoperatively misinterpreted as hemangioma (Figures 3B2, B3 and 5B2, B3).

MRI was performed in five patients (15.6\%) who presented with melena $(\mathrm{n}=1)$, hematochezia $(\mathrm{n}=1)$, abdominal pain $(\mathrm{n}=1)$, and abdominal mass $(\mathrm{n}=1)$ and was also performed as an incidental imaging procedure $(n=1)$. MRI provided a provisional diagnosis of a mesenchymal tumor of the abdominal or pelvic cavity in the five patients, but it did not identify the site of origin of the primary lesion (Figures 1B1-B3 and 2A3, A4). 

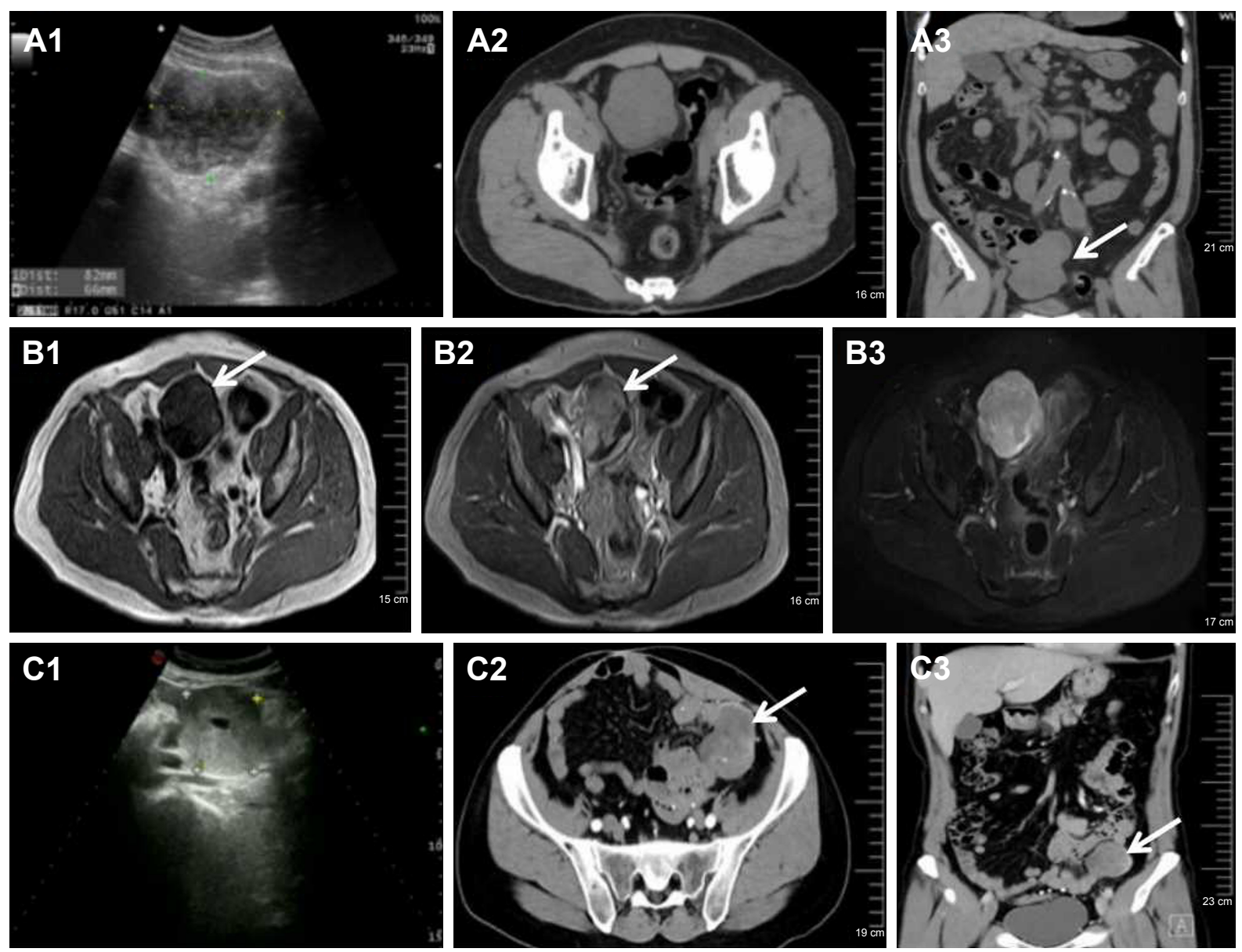

Figure I Gastrointestinal stromal tumors arising from the ileum.

Notes: (AI-B3) A 63-year-old male presenting with hematochezia. (AI) Transabdominal ultrasound image shows a $6.6 \times 8.2 \mathrm{~cm}^{2}$ well-defined, heterogeneous and hypoechoic mass above the urinary bladder. (A2) Axial plain CT image shows a soft tissue mass located in the pelvis. (A3) Coronal plain CT image shows a lobulated pelvic mass (arrow). (BI) Axial TIWI shows an isodense mass (arrow). (B2) Axial contrast-enhanced TIWI shows the mass with markedly heterogeneous enhancement (arrow). (B3) Axial T2WI showing the mass with heterogeneous hyperintensity. (CI-C3) A 48-year-old male presenting with abdominal mass. (CI) Transabdominal ultrasound image shows a $5.2 \times 5.7 \mathrm{~cm}^{2}$ well-defined, heterogeneous and hypoechoic mass in the left lower abdomen. (C2) Axial contrast-enhanced CT image shows an irregular mass with markedly heterogeneous enhancement (arrow). (C3) Coronal contrast-enhanced CT image shows an exophytic mass arising from small bowel with inhomogeneous enhancement (arrow).

Abbreviations: CT, computed tomography; WI, weighted imaging.

Both EGD and colonoscopy were performed in 16 patients (50\%) who presented with GI bleeding (melena and/or hematochezia), but no hemorrhagic lesion was identified in the esophagus, stomach, duodenum (the first and second parts), colon, or rectum.

CE was performed in two patients $(6.2 \%)$ who presented with intermittent melena and in whom both EGD and colonoscopy findings were normal. In the first case (Figure 5A), an indistinct mass and dark red blood clot were identified in the jejunum with $\mathrm{CE}$, but the source of blood loss remained unidentified. DBE was requested, which showed a well-circumscribed tumor measuring $2.5 \times 2.5 \mathrm{~cm}^{2}$ with umbilication and blood clot in the middle part of the jejunum. In the second case that presented with intermittent melena (Figure 5B), CTA identified a round tumor measuring $1.5 \times 1.6 \mathrm{~cm}^{2}$ with both intraluminal and exophytic components and an enhanced vascular pattern in the jejunum. A protruding lesion was identified in the upper part of the jejunum with CE and DBE. Subsequent laparoscopic surgery identified the tumor in the jejunum at $\sim 30 \mathrm{~cm}$ from the ligament of Treitz.

Double-balloon enteroscopy (DBE) was performed in nine patients $(28.1 \%)$ who presented with GI bleeding, and EGD and colonoscopy findings were unremarkable. Review of the imaging findings of the 32 cases included in the study showed the DBE located the lesion in the small bowel in eight out of nine cases $(88.9 \%)$ of small bowel GIST. The tumors were located in the third part of the 

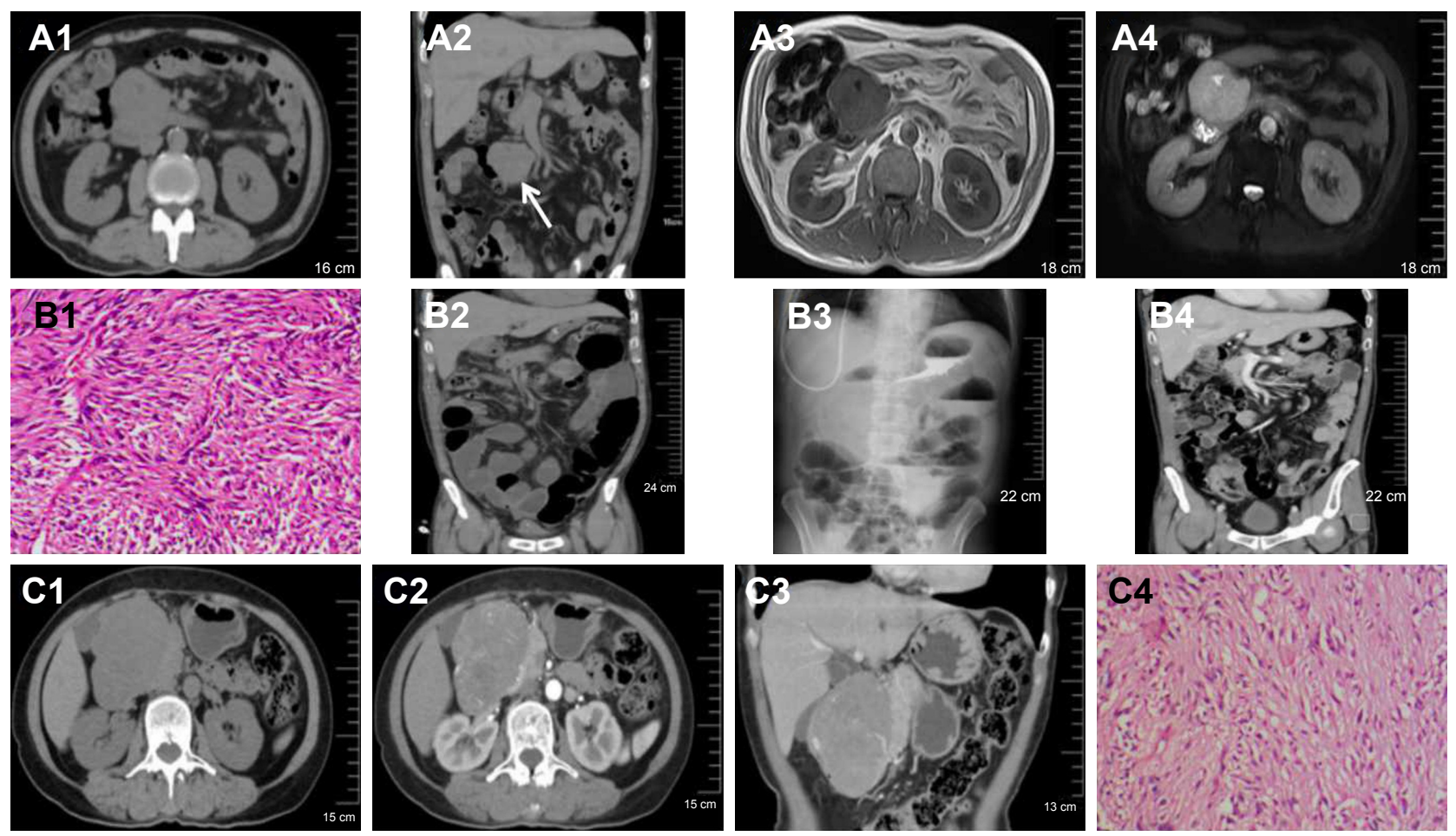

Figure 2 Gastrointestinal stromal tumors arising from the duodenum.

Notes: (AI-B4) A 60-year-old male with an incidentally abdominal space occupying lesion during routine checkup. (AI) Axial plain $\mathrm{CT}$ image shows a $5.1 \times 5.2 \mathrm{~cm}^{2}$ slightly heterogeneous mass. (A2) Coronal plain CT image shows a well-defined, irregular mass (arrow). (A3) Axial TIWI shows the mass with slight hyperintensity. (A4) Axial T2Wl shows the mass with heterogeneous hyperintensity. (BI) Histopathology shows the tumor cells are composed of spindle cells with a high mitotic count ( $>5$ mitoses/50 HPFs; HE staining; original magnification $\times 100$ ). (B2) A week after the operation, repeated CT scanning demonstrates the patient complicated with intestinal obstruction. (B3) Ten days after the operation, abdominal plain X-ray reveals the patient still accompanied with partial intestinal obstruction. (B4) The mass was resected, and the patient was started on adjuvant imatinib. Follow-up contrast-enhanced CT scanning 10 months after treatment, shows that there is no local recurrence and distant metastasis. (CI-C4) A 56-year-old female presenting with abdominal mass. (CI) Axial plain CT image shows a $6.7 \times 8.7 \mathrm{~cm}^{2}$ mass in the right upper quadrant of the abdomen. (C2) Axial contrast-enhanced CT image shows the mass with markedly heterogeneous enhancement. (C3) Coronal contrast-enhanced CT image shows a well-defined, oval mass with inhomogeneous enhancement, but suggestive of a pancreatic tumor. (C4) Histopathology shows the tumor cells are composed by mixed spindle and epithelioid cells with a low mitotic count ( $\leq 5$ mitoses/50 HPFs; HE staining; original magnification $\times 100$ ).

Abbreviations: CT, computed tomography; WI, weighted imaging; HPFs, high-power fields; HE, hematoxylin and eosin.

duodenum (n=1; Figure 4A3), the upper part of the jejunum $(\mathrm{n}=2$; Figure 5B5, C5), the middle part of the jejunum ( $\mathrm{n}=4$; Figures $4 \mathrm{~B} 3$ and $5 \mathrm{~A} 4)$, and the upper part of the ileum $(\mathrm{n}=1$; Figure 6A3). DBE did not show the ninth lesion as it was with exophytic growth (Figure 4C), but a protrusion was identified in the upper part of the jejunum. The patient who presented with recurrent hematochezia underwent an emergency laparotomy, and during surgery, a well-circumscribed mass, measuring $2.5 \times 3 \mathrm{~cm}^{2}$, was identified at the jejunum located $10 \mathrm{~cm}$ from the ligament of Treitz.

Exploratory laparotomy was performed in one patient (3.1\%) who was identified as having a small bowel tumor on imaging, who had presented clinically with acute abdominal pain and ascites, with small bowel obstruction confirmed on CT imaging. Laparotomy showed small bowel volvulus and ischemia associated with symptoms of septic shock. Subsequently, a $4.0 \times 4.5 \mathrm{~cm}^{2}$ diameter mass with exophytic growth was identified in the jejunum located $20 \mathrm{~cm}$ from the ligament of Treitz.

\section{Histopathology and immunohistochemical findings in 32 cases of primary GIST of the small bowel}

Table 2 summarizes the histopathologic features of the 32 cases of small bowel GIST included in this study. The GIST was located in the duodenum in 4 patients (12.5\%), in the jejunum in 21 patients $(65.6 \%)$, and in the ileum in 7 patients $(21.9 \%)$. The mean diameter of the 32 cases of small bowel GIST was $5.3 \mathrm{~cm}$ (range, $1-14 \mathrm{~cm}$ ). There were 17 patients (53.1\%) with exophytic tumors (Figure 4C), and the tumors were intraluminal in 6 patients (18.8\%; Figure $5 \mathrm{~A})$, and 9 patients $(28.1 \%)$ had GIST with both exophytic and intraluminal components (Figures 4B and 6A). Tumors had well-circumscribed margins in 26 patients $(81.2 \%)$ and 

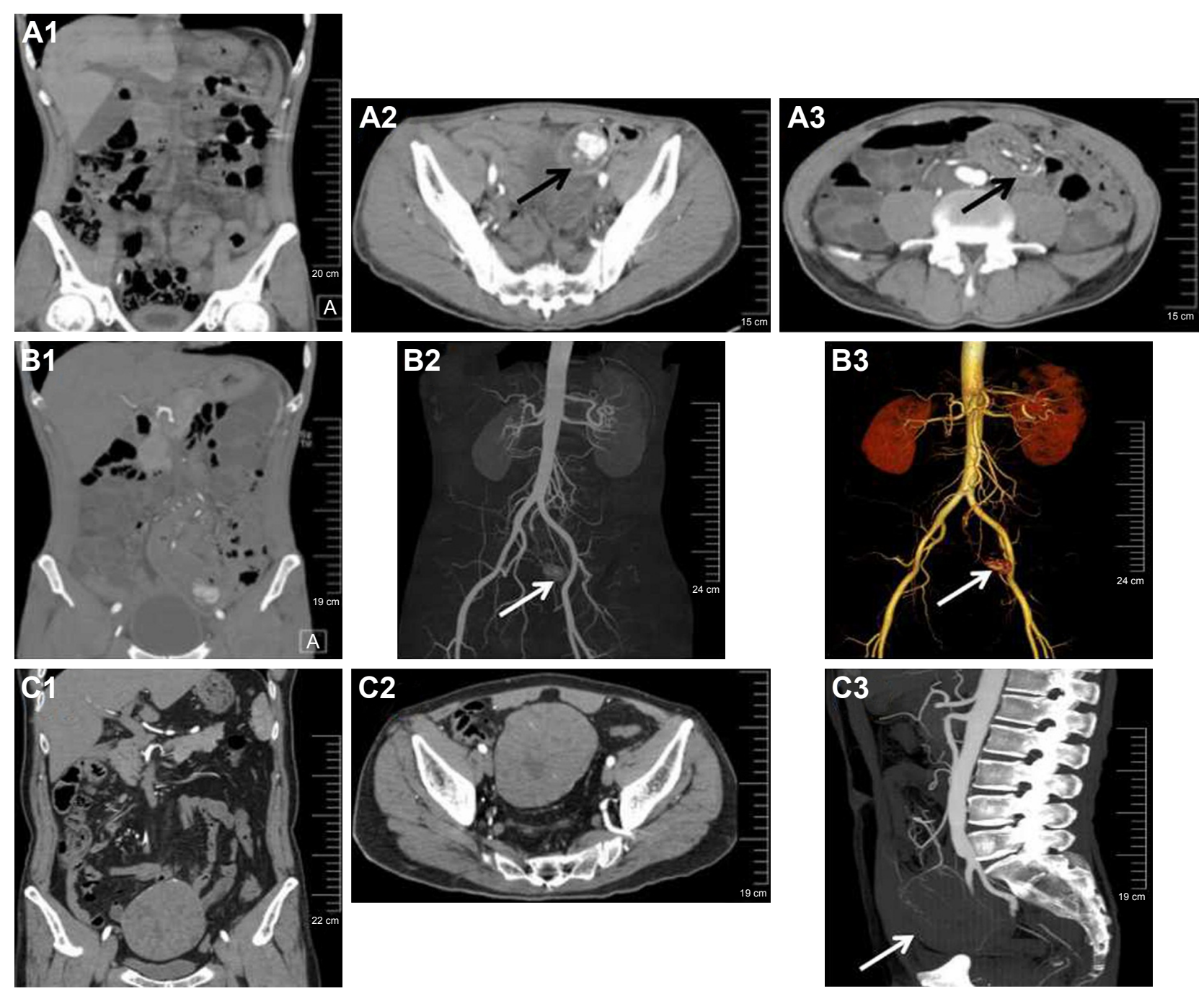

Figure 3 SB GISTs-associated abdominal pain and distension.

Notes: (AI-B3) A 49-year-old male presenting with abdominal pain. (AI) Coronal plain CT image shows the patient with partial intestinal obstruction on admission, but no primary tumor is found. (A2) Five days after admission, axial CTA image shows a $1.6 \times 2.5 \mathrm{~cm}^{2}$ well-circumscribed intraluminal tumor with intense enhancement (arrow). (A3) In the above level, intussusception of the small bowel is seen (arrow). (BI) Coronal CTA image shows bowel wall edema and thickening with the intraluminal tumor. (B2) Coronal CTA image shows the feeding artery of the tumor (arrow). (B3) 3D reconstruction of CTA image shows the tumor (arrow) is supplied by branch vessels from the superior mesenteric artery. (CI-C3) A 57-year-old male presenting with abdominal distension. (CI) Coronal CTA image shows a pelvic mass in close association with the bowel loops, confirmed to be arising from the ileum at surgery. (C2) Axial CTA image shows an $8.0 \times 10.0 \mathrm{~cm}^{2}$ well-defined mass with smooth outline. (C3) Sagittal CTA image shows the feeding artery of the tumor (arrow) from the branch of superior mesenteric artery.

Abbreviations: SB GISTs, small bowel gastrointestinal stromal tumors; CT, computed tomography; CTA, computed tomography angiography.

ill-defined margins in 6 patients $(18.8 \%)$. The tumors were smooth in outline in 24 patients $(75 \%)$, while they were irregular in outline in 8 patients $(25 \%)$.

Histologically, 24 tumors (75\%) were composed of spindle cells (Figures $2 \mathrm{~B} 1$ and $6 \mathrm{~B} 1$ ) and 8 tumors (25\%) were mixed, containing both spindle cells and epithelioid cells (Figure 2C4). No purely epithelioid cell tumors were found in this case series. Mitoses were detected in the small bowel GIST tissues, with 22 tumors $(68.8 \%)$ having a mitotic count $\leq 5$ per 50 HPFs and 10 tumors (31.2\%) having a mitotic count $>5$ per 50 per HPFs. Based on the tumor size and mitotic index, 5 tumors $(15.6 \%)$ were classified as very low risk, 15 tumors $(46.9 \%)$ as low risk, $0(0 \%)$ as intermediate risk, and 12 tumors (37.5\%) were classified as high risk, according to the modified NIH classification system. ${ }^{11}$

Necrosis was found in 6 tumors $(18.8 \%)$, ulceration or erosion in 12 tumors (37.5\%), and local adhesion or invasion into adjacent organs in 9 tumors (28.1\%; Figure 4B4), but none of the patients had tumor metastasis at the time of diagnosis.

Immunohistochemistry showed that all $32(100 \%)$ of the primary small bowel GIST included in this study were CD117 positive (Figure 6B2), 26 cases (81.2\%) were CD34 positive (Figure 6B3), and 30 cases (93.8\%) were DOG1positive (Figure 6B4), whereas 20 cases $(62.5 \%)$ were 

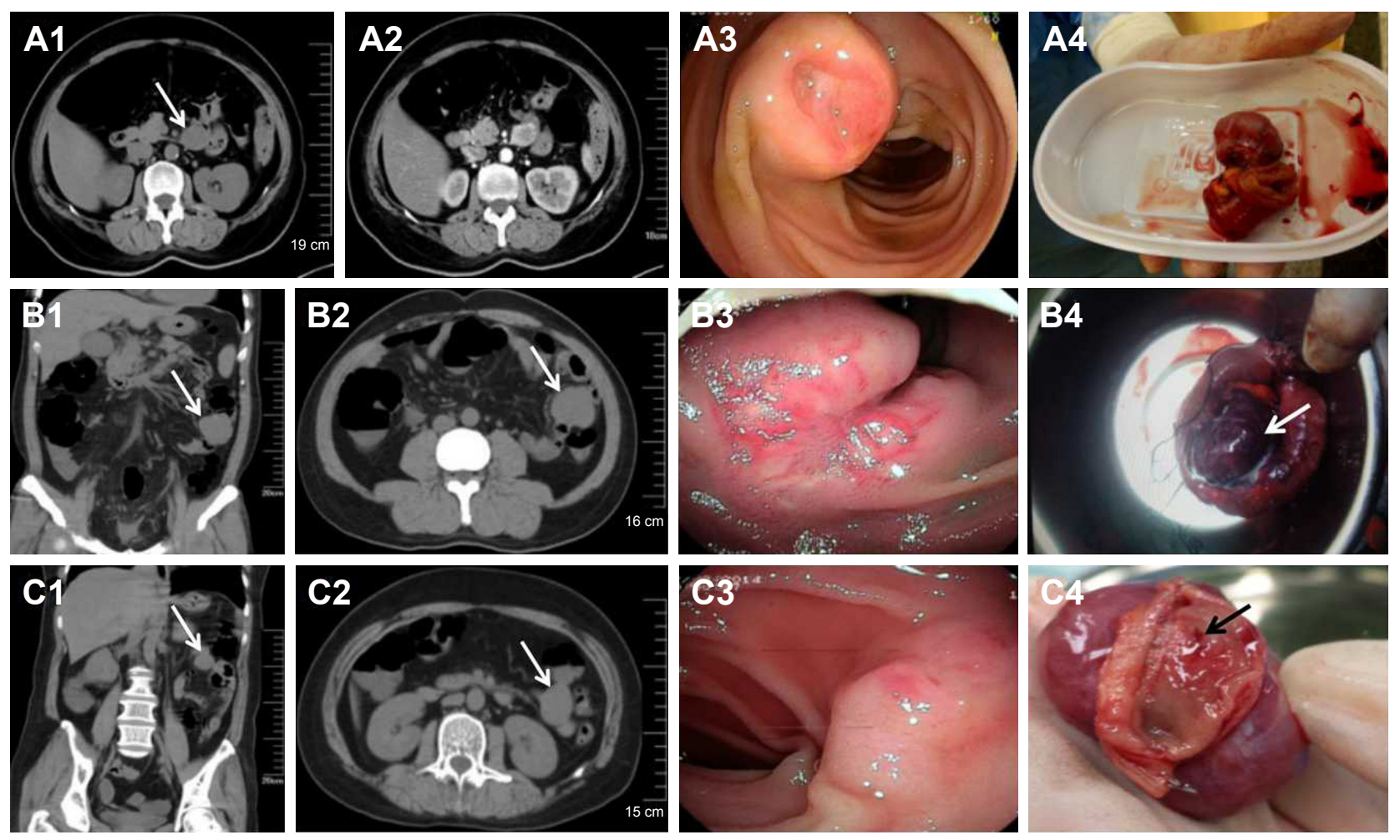

Figure 4 SB GISTs-associated gastrointestinal bleeding.

Notes: (AI-A4) A 57-year-old female with a duodenal GISTs presented with melena. (AI) Axial plain CT image shows a $2.0 \times 2.2 \mathrm{~cm}^{2}$ well-defined, isodense mass (arrow). (A2) Axial contrast-enhanced CT image shows the mass with markedly heterogeneous enhancement in the duodenum (3rd part). (A3) DBE shows a round tumor with central ulceration. (A4) Macroscopic appearance of the surgical specimen. (BI-B4) A 46-year-old male with a jejunal GISTs presented with melena. (BI) Coronal plain CT image shows a $3.5 \times 4.0 \mathrm{~cm}^{2}$ isodense mass (arrow) arising from small bowel. (B2) Axial plain CT image shows the mass (arrow) with extraluminal and intraluminal components. (B3) DBE shows a tumor with engorged vessels and deep ulcer in the jejunum (middle part). (B4) Gross appearance of the operative specimen shows adherence of the adjacent bowel to the tumor (arrow). (CI-C4) A 56-year-old female with a jejunal GISTs presented with hematochezia. (CI) Coronal plain CT image shows an exophytic mass (arrow) arising from the jejunum. (C2) Axial plain CT image shows a $2.5 \times 3.0 \mathrm{~cm}^{2}$ well-defined, round mass (arrow). (C3) DBE shows a protrusion with mucosal erosion in the jejunum (upper part). (C4) Gross appearance of the operative specimen shows an exophytic tumor with the bleeding point (arrow), indicative of the bleeding source.

Abbreviations: SB GISTs, small bowel gastrointestinal stromal tumors; CT, computed tomography; DBE, double-balloon enteroscopy.

SMA-negative (Figure 6C1), 29 cases $(90.6 \%)$ were S100negative (Figure 6C2), and 30 cases $(93.8 \%)$ were desminnegative (Figure 6C3). The cutoff between a low and a high proliferation index of Ki- 67 was accepted as $10 \%$, with 28 cases (87.5\%) having a low Ki-67 index $(<10 \%$; Figure $6 \mathrm{C} 4)$ and 4 cases (12.5\%) having a high Ki-67 index $(\geq 10 \%)$.

\section{Surgical management and clinical outcome in 32 patients diagnosed with primary GIST of the small bowel}

The primary tumor was resected in all 32 patients. Elective surgery was scheduled for 29 patients $(90.6 \%)$, and for the other 3 patients $(9.4 \%)$, emergency surgery was performed due to active GI bleeding or intestinal obstruction. There were 25 patients $(78.1 \%)$ who underwent open surgery, while the other 7 patients (21.9\%) underwent laparoscopic surgery. Among the 32 patients in the study, 27 patients (84.4\%) underwent segmental resection of the small bowel with the removal of the tumor and the other contiguous organs involved. Two patients $(6.2 \%)$ with a tumor in the duodenum underwent pancreaticoduodenectomy and the other three patients $(9.4 \%)$ with isolated tumors underwent local excision. All of the 32 patients in the case series (100\%) achieved an R0 surgical resection result.

Postoperative complications, including pancreatic leakage, occurred in two cases, and intestinal obstruction occurred in two cases (Figure 2B2 and B3), liver dysfunction in one case, and pulmonary infection in two cases, which all resolved following conservative treatment, as shown in Table 3. There were no cases of postoperative mortality in this case series, and the postoperative hospital stay ranged from between 7 and 32 days (mean, 14.4 days).

\section{Patient follow-up}

The median follow-up period was 30 months (range, 3-54 months; Table 4). Of the 32 patients who underwent complete tumor resection, local recurrence on follow-up imaging was found in none of them (Figure 2B4). One high-risk patient 

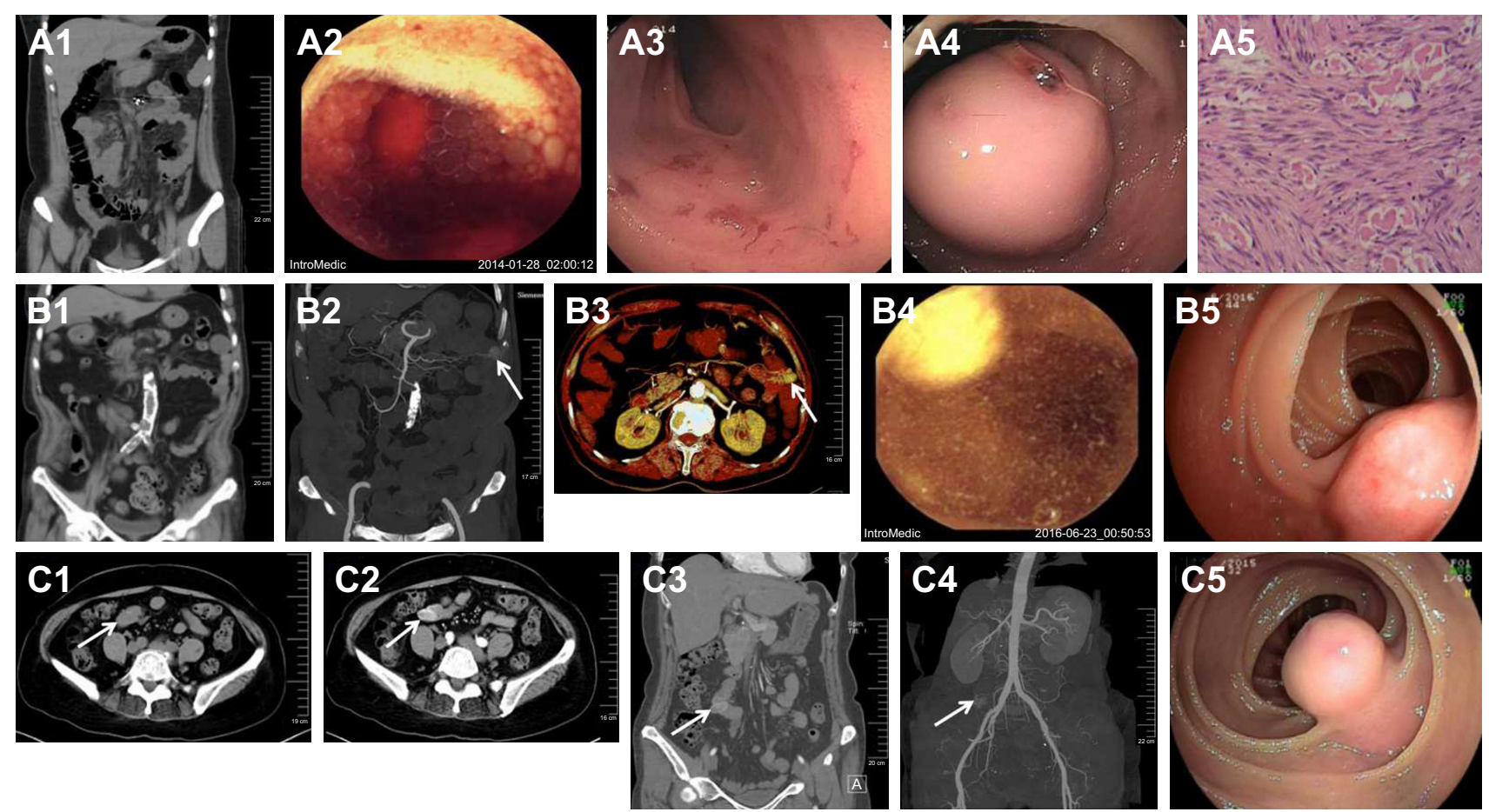

Figure 5 SB GISTs-associated gastrointestinal bleeding.

Notes: (AI-A5) A 23-year-old female with a jejunal GISTs presented with melena. (AI) Coronal plain CT image does not reveal the primary tumor due to poor bowel preparation. (A2) CE shows a blurry mass and dark-red blood residue in the jejunum (middle part). (A3) DBE shows the jejunal blood clots. (A4) DBE shows a $2.5 \times 2.5 \mathrm{~cm}^{2}$ round, smooth tumor with umbilication. (A5) Histopathology shows the tumor cells are composed of spindle cells with a low mitotic count ( $\leq 5$ mitoses/50 HPFs; HE staining; original magnification $\times 100$ ). (BI-B5) A 8I-year-old male with a jejunal GISTs presented with melena. (BI) Coronal plain CT image does not reveal the primary tumor due to polymorphous intestine. (B2) Coronal CTA image shows a $1.5 \times 1.6 \mathrm{~cm}^{2}$ well-defined tumor with marked enhancement (arrow), which is supplied by jejunal branch from the superior mesenteric artery. In this case, radiological report suggests a hemangioma. (B3) Axial CTA image shows the tumor (arrow) with extraluminal and intraluminal components. (B4) CE shows a protruded lesion in the jejunum (upper part). (B5) DBE shows the intraluminal component of the tumor with mucosal erosion. (CI-C5) A 58-year-old female with a jejunal GISTs presented with melena. (CI) Axial plain CT image seems to reveal an intestinal soft tissue mass (arrow). (C2) Axial contrast-enhanced CT shows a $1.0 \times 2.2 \mathrm{~cm}^{2}$ tumor with a dumbbell-like appearance and with marked enhancement (arrow). (C3) Coronal CTA image shows a small bowel tumor with markedly peripheral enhancement (arrow). (C4) Coronal CTA image shows the tumor (arrow) is supplied by vessels from the superior mesenteric and iliac arterial territories. (C5) DBE shows a round, smooth tumor in the jejunum (upper part).

Abbreviations: SB GISTs, small bowel gastrointestinal stromal tumors; CT, computed tomography; CTA, computed tomography angiography; CE, capsule endoscopy; DBE, double-balloon enteroscopy; HPFs, high-power fields; HE, hematoxylin and eosin.

(3.1\%) later developed extensive intra-abdominal metastases and died at 18 months postoperatively. One low-risk patient (3.1\%) died due to viral hepatitis-induced acute liver failure, 12 months postoperatively. However, 30 patients $(93.8 \%)$ remained alive until their last clinical follow-up in December 2017.

Only four high-risk patients underwent treatment with imatinib therapy after surgery and were treated for a median time of 19.5 months (range, 6-27 months). All four patients were treated with imatinib at a daily dose of $400 \mathrm{mg}$, which resulted in one patient developing abnormal liver function and another patient developing acute agranulocytosis. The remaining high-risk cases refused imatinib therapy, mainly due to the high cost of this treatment, which was not covered by health insurance or private funding.

\section{Discussion}

GIST is the most common primary mesenchymal tumor of the digestive tract, and although it may arise at any site, $\sim 30 \%-40 \%$ of cases occur in the small bowel..$^{5}$ In this study, a retrospective review of 32 cases of small bowel GIST diagnosed and surgically resected during a 5 -year period at a single center in Wuhan, China has been presented, including their clinical presentation, diagnosis, treatment, and clinical follow-up.

In previously published studies, GIST of the small bowel has been reported to show a slight male predominance. ${ }^{10,12,13}$ However, the findings from this study showed there were equal numbers of men and women, which may have been due to the small study sample size. However, the median age of the patients in the present study was 56 years, which was similar to previously reported case series. ${ }^{12,13}$

The clinical symptoms associated with small bowel GIST are usually nonspecific and varied and are usually associated with tumor size and anatomical site..$^{10,13}$ The majority of patients $(80 \%-88 \%)$ are symptomatic at presentation, with the most common presenting symptoms being GI bleeding, abdominal pain or discomfort, and the presence of an abdominal mass. ${ }^{10,13}$ 

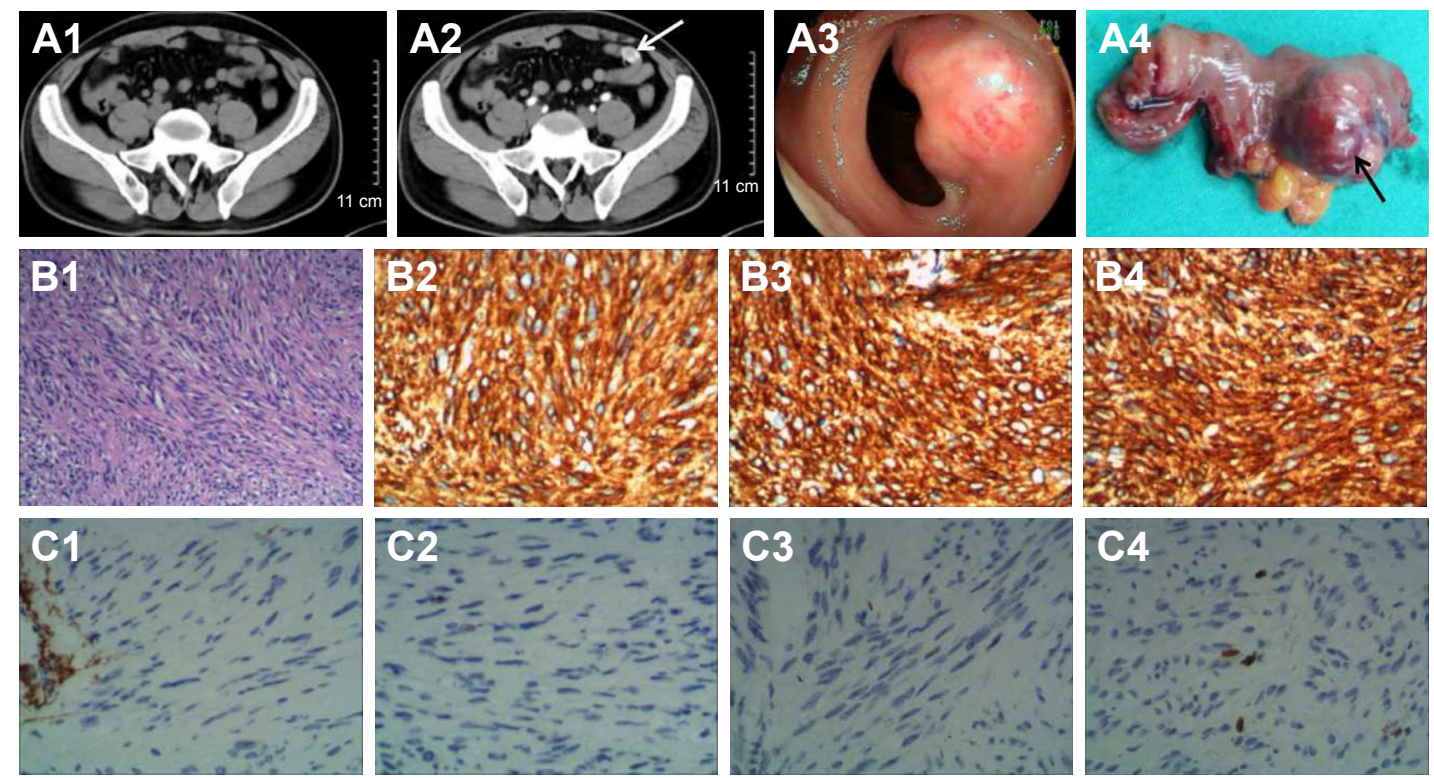

Figure 6 lleal GISTs and immunohistochemistry.

Notes: A 55-year-old male with an ileal GISTs presented with melena. (AI) Axial plain CT image does not reveal the primary tumor due to polymorphous intestine. (A2) Axial contrast-enhanced CT image shows a $1.3 \times 1.8 \mathrm{~cm}^{2}$ tumor (arrow) with marked enhancement in the arterial phase. (A3) DBE shows the intraluminal component of the tumor with engorged vessels. (A4) Gross appearance of the operative specimen shows the extraluminal component of the tumor (arrow). (B I) Histopathology shows the tumor cells are composed of spindle cells with a low mitotic count ( $\leq 5$ mitoses/50 HPFs; HE staining). Tumor cells are strongly positive for CDII7 (B2), CD34 (B3), and DOGI (B4). Tumor cells are negative for SMA (CI), which is positive in vascular wall. S-I00 (C2) and Desmin (C3) are negative in tumor cells. Ki-67 (C4) is positive in some of the tumor nuclei (global index 2\%). (Original magnification: B I $\times 100$; B2-C4 $\times 200$ ).

Abbreviations: GISTs, gastrointestinal stromal tumors; CT, computed tomography; DBE, double-balloon enteroscopy; HPFs, high-power fields; HE, hematoxylin and eosin; SMA, smooth muscle actin.

Other relatively rare presentations of small bowel GIST include anemia, intraperitoneal hemorrhage, peritonitis, and altered bowel motility. ${ }^{13-15}$ Between $12 \%$ and $18 \%$ of patients with small bowel GIST have been reported to be asymptomatic, with the tumors detected incidentally., ${ }^{3,13}$

In this case series of 32 patients with histologically confirmed primary small bowel GIST, 3 patients $(9.4 \%)$ did not present with clinical symptoms and their tumors were discovered incidentally on CT scan. In 15 patients (46.8\%), GI bleeding was the more common presenting symptom, and in 9 patients (28.2\%), abdominal pain and distension were the symptoms. These findings were similar to those from a previously published report from Taiwan. ${ }^{12}$ Clinically, GI bleeding associated with primary GIST of the small bowel
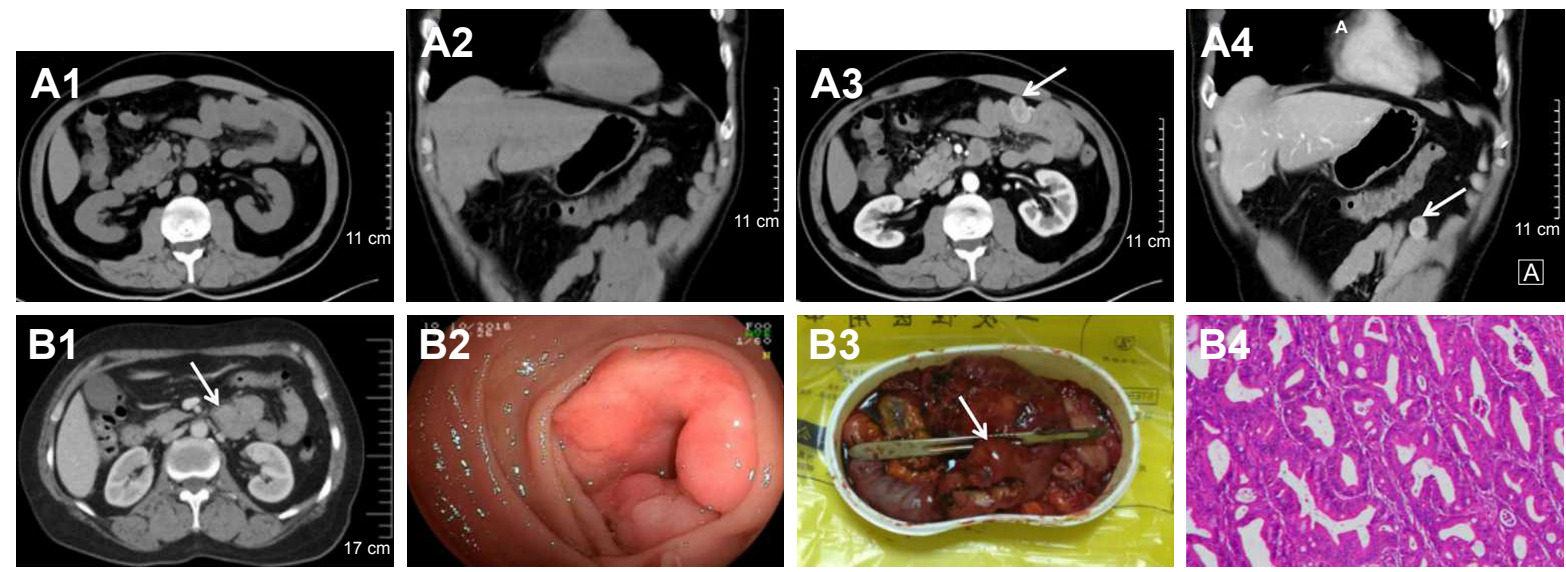

Figure 7 Jejunal GISTs and duodenal adenocarcinoma.

Notes: (AI-A4) A 54-year-old male with an incidentally jejunal GISTs during CT scanning for acute diarrhea. Axial (AI) and coronal (A2) plain CT images do not reveal the primary tumor due to intestine loops overlaps. Axial (A3) and coronal (A4) contrast-enhanced CT images show a $1.8 \times 2.0 \mathrm{~cm}^{2}$ exophytic tumor (arrow) with marked enhancement, but suggestive of a hemangioma. (B I-B4) A 57-year-old female with a duodenal adenocarcinoma presented with abdominal pain and vomiting. (BI) Axial contrast-enhanced CT image shows a $3.0 \times 4.5 \mathrm{~cm}^{2}$ tumor (arrow) with heterogeneous enhancement in the duodenum (4th part), but suggestive of a GISTs. (B2) DBE shows the intraluminal part of the tumor with obvious lumen stenosis. (B3) Macroscopic appearance of the surgical specimen. (B4) Histopathology shows the tumor to be a moderately poorly differentiated adenocarcinoma (HE staining; original magnification $\times 100$ ).

Abbreviations: GISTs, gastrointestinal stromal tumors; CT, computed tomography; DBE, double-balloon enteroscopy; HE, hematoxylin and eosin. 
Table 2 Summary of the major macroscopic and histopathology characteristics of the 32 cases of small bowel GIST

\begin{tabular}{|c|c|}
\hline Variable & Results \\
\hline \multicolumn{2}{|l|}{ Location } \\
\hline Duodenum vs jejunum vs ileum & 4 vs 21 vs 7 \\
\hline \multicolumn{2}{|l|}{ Tumor size (maximal diameter, $\mathrm{cm}$ ) } \\
\hline Range & $1-14$ \\
\hline Mean & 5.3 \\
\hline \multicolumn{2}{|l|}{ Tumor size $(\mathrm{cm})$} \\
\hline$\leq 5$ vs $5-10$ vs $\geq 10$ & 20 vs 8 vs 4 \\
\hline \multicolumn{2}{|l|}{ Growth pattern } \\
\hline Exophytic vs intraluminal vs combined & 17 vs 6 vs 9 \\
\hline \multicolumn{2}{|l|}{ Tumor margin } \\
\hline Well circumscribed vs ill-defined & 26 vs 6 \\
\hline \multicolumn{2}{|l|}{ Tumor outline } \\
\hline Smooth/mildly lobulated vs irregular & 24 vs 8 \\
\hline \multicolumn{2}{|l|}{ Cell type } \\
\hline Spindle vs epithelioid vs mixed & 24 vs 0 vs 8 \\
\hline \multicolumn{2}{|l|}{ Mitotic count (in 50 HPFs) } \\
\hline$\leq 5$ vs $>5$ & 22 vs 10 \\
\hline \multicolumn{2}{|l|}{ NIH risk categories } \\
\hline Very low vs low vs intermediate vs high & 5 vs 15 vs 0 vs 12 \\
\hline \multicolumn{2}{|l|}{ Necrosis } \\
\hline Absence vs presence & 26 vs 6 \\
\hline \multicolumn{2}{|l|}{ Ulceration or erosion } \\
\hline Absence vs presence & 20 vs 12 \\
\hline \multicolumn{2}{|l|}{ Local adhesion or invasion to adjacent organs } \\
\hline Absence vs presence & 23 vs 9 \\
\hline \multicolumn{2}{|l|}{ Metastasis } \\
\hline Absence vs presence & 32 vs 0 \\
\hline
\end{tabular}

Abbreviations: GIST, gastrointestinal stromal tumor; HPFs, high power fields; $\mathrm{NIH}$, National Institutes of Health.

Table 3 Operative characteristics and postoperative outcomes of the 32 patients studied

\begin{tabular}{ll}
\hline Variable & Results \\
\hline Operation time & 3 \\
Emergent & 29 \\
Elective & \\
Operative procedure & 25 \\
$\quad$ Open surgery & 7 \\
Laparoscopic surgery & \\
Operation type & 2 \\
$\quad$ Pancreaticoduodenectomy & 3 \\
Localized resection & 27 \\
Segmental intestinal resection & \\
Margins status & 32 \\
R0 & 0 \\
RI & 0 \\
R2 & \\
Postoperative complications & 2 \\
Pancreatic leakage & 2 \\
Intestinal obstruction & 1 \\
Elevation of transaminase & 2 \\
Pulmonary infection & 0 \\
Surgery-related death & \\
Postoperative hospital stay (days) & $7-32$ \\
Range & 14.4 \\
Mean &
\end{tabular}

Table 4 The clinical follow-up of the 32 patients studied

\begin{tabular}{ll}
\hline Variable & Results \\
\hline $\begin{array}{l}\text { Follow-up (months) } \\
\quad \text { Range }\end{array}$ & $3-54$ \\
$\quad$ Median & 30 \\
Postoperative adjuvant therapy & \\
None & 28 \\
Alive vs dead & 26 vs 2 \\
Imatinib & 4 \\
Alive vs dead & 4 vs 0 \\
Tumor recurrence & 0 \\
Tumor metastasis & $\mathrm{I}$ \\
\hline
\end{tabular}

usually arises from the ulcerated or necrotic component of the tumor (Figures 4A, B and 5A).

The presenting symptoms of abdominal pain and distension in patients with small bowel GIST may be the result of the exophytic and intraluminal growth of the tumor, resulting in intestinal obstruction or intussusception (Figure 3A), or because of the effects of an exophytic tumor compressing the bowel lumen (Figure 3C). Also, exophytic tumors may present as a palpable mass (Figures $1 \mathrm{C}$ and $2 \mathrm{C}$ ).

The preoperative diagnosis of primary GIST of the small bowel is difficult to make due to the relative inaccessibility of the small bowel to conventional endoscopic examination. Also, it can be difficult to determine the nature of the mass solely on imaging of the abdomen. In the present study, alternative imaging diagnostic modalities for small bowel GIST included ultrasound, CT, CTA, and MRI. An abdominal ultrasound scan is often the initial imaging test employed in the investigation of a patient with abdominal pain or mass, but ultrasound is not an accurate method for the detection of tumors of the small bowel and has been reported to have a low sensitivity of no more than $26 \% .{ }^{16}$ Because of the availability of CT imaging, this method is often used for diagnosing small bowel GIST and can be used for the detection, localization, staging, surgical planning, and in the evaluation of response to therapy, including monitoring postoperative follow-up in patients with GIST ${ }^{8,17}$ In 12 patients with primary GIST of the small bowel, Nakatani et al observed that the detection rate of CT was $67 \% .{ }^{18}$ Recently, the use of CTA in the diagnosis of small bowel tumors with GI bleeding has become feasible, with a reported diagnostic sensitivity of $90.9 \%$ for small bowel GIST. ${ }^{19,20}$ MRI offers good soft tissue contrast and is also an effective imaging modality when differentiating small bowel GIST from other small bowel tumors or masses. ${ }^{21,22}$

In the present study, the provisional diagnosis of small bowel GIST was made by ultrasound in 0 out of 9 cases, by 
CT in 17 out of 31 cases, by CTA in 5 out of 7 cases, and by MRI in 0 out of 5 cases. These varied imaging findings may have been influenced by several factors in this study. Some tumors of small size were less likely to be detected on imaging, or the tumor might have been covered by overlapping loops of small bowel, or there may have been poor bowel preparation prior to imaging (Figures 5A1, B1, 6A1, and 7A1, A2). However, although imaging studies might be better able to detect large lesions, which might fill the abdominal or pelvic cavity, and push aside or invade surrounding organs, the site of origin of the tumor may not be identifiable, which may result in small bowel GIST of large size being misdiagnosed on imaging as pancreatic tumors, gynecological tumors, or tumors of the mesentery (Figures 1A, B and 2C). In some patients with small bowel GIST who present with intestinal obstruction or intussusception, as in the present study, the radiologist might have difficulty in identifying the primary lesion and defining its type due to small bowel distention, bowel wall edema and thickening (Figure 3A and B1). There may also be a lack of characteristic features of small bowel GIST that could differentiate this tumor from other possible primary small bowel tumors, such as hemangioma (Figures 3A, B, 5B2, B3, and 7A), lymphangioma, and adenocarcinoma (Figure 7B). A further factor that might result in difficulty in diagnosing small bowel GIST on imaging might include a lack of exchange of key clinical information between gastroenterologists and radiologists, which, when combined with the rare nature of small bowel GIST, might delay the diagnosis.

Four commonly used endoscopic diagnostic procedures used to detect small bowel GIST were used in this study, but not for all patients, and included EGD, colonoscopy, CE, and DBE. However, EGD can be performed up to the second portion of the duodenum and can identify some duodenal tumors, whereas, colonoscopy can only examine the GI tract up to the terminal ileum.

$\mathrm{CE}$ is a safe and painless method for mucosal imaging of the small bowel and can be most useful in patients with obscure GI bleeding. However, lack of air insufflation, rinsing, and the unclear images caused by bowel content or active bleeding can impair the detection of small bowel lesions such as GIST (Figure 5A2 and B4). ${ }^{18,23}$ However, DBE enables endoscopic inspection of the entire small bowel with the ability to take biopsy samples and with the potential to administer localized therapy. A further advantage of DBE is that it allows direct visualization of the small bowel mucosa and confirmation of the diagnosis that might only be suspected with CE or radiologic examination (Figures 4A3,
B3, 5A4, B5, C5, and 6A3). ${ }^{23}$ The positive detection rate of DBE for diagnosing small bowel GIST was $88.9 \%$ in the present study. This result is supported by the previously published findings of He et al, who found that the diagnostic yield of DBE for mesenchymal tumors of the small bowel was $88.3 \% .{ }^{24} \mathrm{~A}$ similar result was reported by Chen et al, who found that the positive detection rate for DBE in the patients with small bowel tumors was $85.9 \% .{ }^{25}$ Also, although small bowel GIST commonly has an exophytic growth pattern (Table 2), some GISTS may still be difficult to detect by endoscopy (Figures 4C3 and 5B4, B5). ${ }^{8,18}$ However, previous studies have found that CT or MRI imaging can be useful for diagnosing small bowel GIST with exophytic growth (Figures 4C1, C2 and 7A) ${ }^{8,18}$ Clinically, some cases of small bowel GIST can cause life-threatening symptoms, including massive GI bleeding and acute small bowel obstruction, and even when endoscopy and imaging had failed to make the diagnosis, emergency exploratory laparotomy can be successful as a diagnostic and treatment procedure..$^{15,26}$

Considering the recent developments and increasing use of other available diagnostic methods, for example, CT enterography, CTA, and magnetic resonance (MR) enterography, a combination of endoscopic (CE and/or DBE) and radiological techniques (CT and/or MRI) might be recommended to ensure earlier and more accurate diagnosis of small bowel GIST in future.

The findings of the present study showed that jejunal GIST (65.6\%) was more common than ileal GIST (21.9\%) and duodenal GIST(12.5\%), which is a finding that is supported by previously published case studies. ${ }^{10,12}$ In the retrospective study of 85 patients with primary small bowel GIST conducted by Wu et al, $61.2 \%$ of the tumors involved the jejunum and the size of the small bowel GIST ranged from 0.3 to $40 \mathrm{~cm}$ in diameter. ${ }^{12}$ The tumor size in the present study ranged from 1.0 to $14.0 \mathrm{~cm}$ in diameter, with a mean diameter of $5.3 \mathrm{~cm}$. A previously published case series by Nakatani et al, which included 12 cases of primary GIST of the small bowel, found a mean tumor diameter of $3.6 \mathrm{~cm}$ (range, $1.4-8.0 \mathrm{~cm}$ ). ${ }^{18}$

In the present study, the cases of small bowel GIST were mainly well circumscribed and with a smooth surface, and were exophytic tumors. These findings are supported by the findings from Baheti et al in their study of 102 cases of small bowel GIST of the jejunum and ileum. ${ }^{13}$ Also, in the present study, necrosis (18.8\%), and mucosal ulceration and erosion were present in $37.5 \%$ of cases of small bowel GIST, which might explain the clinical symptoms of GI bleeding (Figures 4A, B and 5A). 
Tissue histology, supported by immunohistochemistry, is required for the definitive diagnosis of GIST, which can distinguish GIST from other types of soft tissue tumors, including smooth muscle cell tumors, schwannoma, and inflammatory fibroid polyp. ${ }^{27}$ In this study, light microscopy, using H\&E-stained tumor tissue sections showed a spindle cell GIST (75\%) to be the most common histological type, which is consistent with previous reports. ${ }^{12,13}$ The tumor cell mitotic count (or mitotic index) is one of the factors that predict prognosis for small bowel GIST, as this reflects tumor grade and speed of growth. ${ }^{12}$ In the present study, $31 \%$ of the small bowel GISTs showed more than five mitoses per 50 HPFs on light microscopy. This finding was significantly lower than the observation of $\mathrm{Wu}$ et al, who found that $64.7 \%$ of cases of small bowel GISTs had a mitotic index of more than five mitoses per $50 \mathrm{HPFs} .{ }^{12}$ According to the revised tumor risk categories from the NIH classification system, ${ }^{11}$ the proportion of patients with small bowel GIST classified into the high-risk group (37.5\%) in this study was also lower than in previously reported studies. Xing et al studied 197 patients with primary GIST of the small bowel and reported 92 cases (46.7\%) that could be classified as high-risk cases, and between $14 \%$ and $26 \%$ of the patients had metastases at the time of diagnosis, involving the liver, peritoneum, omentum, lung, bone, and kidney. ${ }^{10}$ However, in the 32 cases presented in the current cases series, there were no patients who presented with tumor metastases.

In this study, a preoperative needle biopsy was not undertaken due to the risk of hemorrhage and the possibility of seeding metastases in the case of a potentially malignant tumor. Also, preoperative biopsy is not necessary when the tumor is suggestive of GIST and is considered resectable or operable. ${ }^{29,40}$ When neoadjuvant imatinib therapy is being considered for non-metastatic GIST when R0 resection is not feasible, pre-perative biopsy should be performed. ${ }^{29}$ In this case series, many tumors were hypervascular (Figures $3 \mathrm{C} 3$, 4B3, and 6A3, A4). Therefore preoperative biopsy was not performed, as all 32 cases were considered to be resectable and preoperative imatinib therapy was not used.

In the present study, positive immunohistochemistry staining using primary antibodies for CD117 was $100 \%$, which is a finding that was supported in the previously published study by Wu et al. ${ }^{12}$ In the present study, immunohistochemistry using antibodies for SMA was positive in $37.5 \%$ of cases of GIST of the small bowel, which was similar to the previously reported findings. ${ }^{12,27}$ In the current case series, $9.4 \%$ of cases showed neurogenic differentiation, with positive immunostaining for $\mathrm{S} 100$ protein, which was lower than that reported in the previously published study. ${ }^{12}$ However, in the cases of GIST in the present study, the immunopositivity for CD 34 , DOG1, and desmin was $81.2 \%$, $93.8 \%$, and $6.2 \%$, respectively, which were higher than in previously published studies. ${ }^{12,28}$ In the previous study by $\mathrm{Wu}$ et al, high Ki-67 index (found in 5.9\% of cases) was an independent prognostic factor associated with reduced diseasefree survival for patients with GIST of the small bowel. ${ }^{12}$ In the current case series, $12.5 \%$ of the tumors showed a high Ki-67 index in cases of primary GIST of the small bowel. These histopathologic and immunohistochemical differences may be due to several factors, including sample size, tissue fixation and processing methods, the immunohistochemical methods used, the tumor size and grade of the tumor, genetics, race, or other unknown factors. These varied, but important, findings in terms of patient prognosis indicate the need for further studies on prognostic factors in primary GIST of the small bowel.

Surgery is the standard treatment for non-metastatic GIST. ${ }^{27}$ The main objectives of surgical treatment are to achieve negative surgical resection margins (R0) and to resect the tumor without causing tumor rupture. ${ }^{27,29} \mathrm{~A}$ complete en bloc resection is recommended, whenever feasible, in cases where contiguous organs are involved. ${ }^{5,27}$ Lymphadenectomy is usually not required, since GISTs rarely metastasize to local or regional lymph nodes..$^{29,40,41}$ Surgical resection should be performed with minimal morbidity and, if a multivisceral resection may be required, then multidisciplinary consultation is indicated and preoperative treatment with imatinib should be considered. ${ }^{40,41}$

Complete tumor resection is an important predictor for patient survival rate, according to $\mathrm{Wu}$ et al, who showed that patients with primary GIST of the small bowel who underwent complete resection had a significantly increased median survival of 123.3 months compared with 12.0 months for those who had an incomplete surgical resection. ${ }^{12}$ Surgical procedures include open surgery and laparoscopic resection. Laparoscopic resection is a feasible surgical approach if intraabdominal tumor rupture or seeding is unlikely. ${ }^{27} \mathrm{Ihn}$ et al reported that laparoscopic resection for GIST of the small bowel of $<10 \mathrm{~cm}$ in diameter had a more favorable shortterm postoperative outcome while achieving comparable oncologic results, when compared with open surgery. ${ }^{30}$

Surgery for GIST of the small bowel may include local excision, small intestine segment resection, and pancreatoduodenectomy, with the latter procedure being associated with more serious complications in some patients. ${ }^{31}$ There was no operation-related mortality in the present case series, which 
is a finding supported by the previous study by $\mathrm{Wu}$ et al. ${ }^{12}$ In the present study, a total of 25 patients underwent an open surgical approach and 7 patients underwent laparoscopic resection, with $\mathrm{R} 0$ excision achieved in all of the 32 patients $(100 \%)$. Postoperative complications occurred in seven patients $(21.9 \%)$, there was no postoperative mortality, and the mean postoperative hospital stay was 14.4 days. These results differed from the previous observation by Ihn et al on 95 patients who underwent surgical resection, where 54 patients underwent open surgery and 41 patients underwent laparoscopic surgery for small bowel GIST $\leq 10 \mathrm{~cm}$ in diameter, with 90 patients (94.7\%) achieving R0 resection, 13 patients (13.7\%) having postoperative complications, and 1 patient (1.1\%) who died from postoperative bleeding; the mean duration of postoperative hospital stay was 7.1 days for laparoscopic resection and 12.4 days for open surgery. ${ }^{30}$ These differences may be explained by different patient selection, comorbidities, the experience of the surgeon, or the surgical and hospital facilities.

A laparoscopic surgical approach is clearly discouraged in patients who have large tumors because of the risk of tumor rupture, which is associated with an increased risk for development of intra-abdominal seeding., ${ }^{5,32}$ At a median follow-up of 48 months (range, 1-179 months), Baheti et al reported in their study of 102 patients with GIST of the small bowel patients (including 64 high-risk cases) that 7 patients (7\%) developed local recurrence and 51 patients (50\%) developed metastatic disease. ${ }^{13}$ However, metastatic GIST occurred in only one patient who underwent surgical resection during follow-up in the present study. This difference may be due to the small sample size, shorter follow-up time, and a lower proportion of high-risk cases in our case series.

Imatinib is a first-line standard therapy for unresectable, metastatic, or recurrent GIST, and the standard dosage is $400 \mathrm{mg} /$ day. ${ }^{32,33}$ Surgery is the primary treatment for resectable localized GIST, but $~ 50 \%$ of patients ultimately develop recurrence or metastasis following complete resection. ${ }^{34,35}$ Therefore, the role of imatinib as an adjuvant treatment has been evaluated in several large, randomized, Phase III clinical trials. ${ }^{36-39}$ Data from the ACOSOG Z9001 study showed that adjuvant imatinib therapy (400 $\mathrm{mg}$ daily) for 1 year could significantly prolong the recurrence-free survival (RFS) of patients with primary GIST ( $\geq 3 \mathrm{~cm}$ in size) after complete surgical resection, but improvement in overall survival (OS) was not observed (during median 19.7 months of follow-up). ${ }^{36}$ At a median follow-up of 74 months, the RFS rate was significantly higher in the imatinib treatment arm when compared with placebo and the OS was not significantly different between the imatinib and placebo treatment arms. ${ }^{37}$ The SSGX-VIII/AIO study compared 12 and 36 months of adjuvant imatinib treatment after resection of GIST in patients with a high risk of recurrence, in accordance with the revised NIH tumor risk categories. ${ }^{11}$ At a median follow-up duration of 54 months, a significant improvement was observed in terms of both RFS (5-year RFS: $65.6 \%$ vs $47.9 \%$ ) and OS (5-year OS: $92.0 \%$ vs $81.7 \%$ ) in the 36 -month treatment arm. ${ }^{38}$ In a 2016 follow-up analysis with a median follow-up of 90 months, the 5-year RFS was $71.1 \%$ for 3 years of adjuvant imatinib therapy vs $52.3 \%$ for 1 year of imatinib and the 5 -year OS was $91.9 \%$ vs $85.3 \% .{ }^{39}$ Based on the results of these trials, both Asian and Western guidelines have recommended 3 years of adjuvant treatment with imatinib for patients with highrisk GIST. ${ }^{29,32,40,41}$ Adjuvant treatment in low-risk patients is not indicated. ${ }^{32,40}$ Also, there is insufficient evidence to support adjuvant treatment with imatinib for intermediaterisk patients. ${ }^{29,40,42}$ However, recent studies have found that the longer duration of adjuvant imatinib treatment $(>3$ or $\geq 5$ years) improved the long-term outcomes (5-year RFS and 5-year OS) in Chinese patients with high-risk GIST. ${ }^{43,44}$

However, according to the National Bureau of Statistics of China (http://www.stats.gov.cn/english/), ${ }^{45}$ in 2015, the average per capita disposable income in China was CNY 21,966 and the daily cost of treatment with $400 \mathrm{mg}$ of imatinib was CNY 72,000, which is not covered by the Medicare payment system in China. Cost was the main reason why only four high-risk patients in this study were treated with imatinib therapy after surgery, as the remaining highrisk cases refused to receive imatinib therapy mainly due to economic reasons.

This study had several limitations, including the small study size (32 patients) and the fact that the study was undertaken at a single center, which may have introduced some study bias. Also, this was a retrospective study that relied on the accuracy of clinical records, surgical records, and records of laboratory investigations. In this retrospective, small study population, single-center study, and potential confounding factors were not evaluated.

\section{Conclusion}

The findings of this retrospective review of 32 cases of primary GIST of the small bowel at a single center showed that most cases were symptomatic at presentation and the most frequent presenting symptom was GI bleeding. In this case series, there were equal numbers of men and women. Clinical diagnosis was supported by the endoscopic 
techniques of $\mathrm{CE}$ and $\mathrm{DBE}$ and by imaging techniques, including $\mathrm{CT}$ and MRI, with the definitive diagnosis made by histopathology and immunohistochemistry. Surgical resection was the first-line treatment for patients with primary localized and resectable small bowel GIST. To delay or prevent relapse and prolong patient survival, adjuvant imatinib therapy is indicated in patients with high-risk small bowel GIST, following complete surgical resection and is recommended for 3 years. This retrospective case series study was supported by a review of the literature of the current status of diagnosis, management, and clinical outcome for patients with primary GIST of the small bowel, with the aim of contributing to knowledge of the therapeutic approach and improvement of prognosis in patients who present with primary GIST of the small bowel.

\section{Acknowledgment}

This study was supported by grants from the Nature Science Foundation of Hubei Province (Ref: 2015CFB730).

\section{Author contributions}

SS and JW designed the research. LZ, YL, and JY performed the research. JY, HZ, and XW analyzed the data. LZ, YL, and SS wrote the paper. All authors contributed toward data analysis, drafting and critically revising the paper and agree to be accountable for all aspects of the work. All authors reviewed and approved the final draft of the manuscript.

\section{Disclosure}

The authors report no conflicts of interest in this work.

\section{References}

1. Kindblom LG, Remotti HE, Aldenborg F, Meis-Kindblom JM. Gastrointestinal pacemaker cell tumor (GIPACT): gastrointestinal stromal tumors show phenotypic characteristics of the interstitial cells of Cajal. Am J Pathol. 1998;152(5):1259-1269.

2. Tryggvason G, Gíslason HG, Magnússon MK, Jónasson JG. Gastrointestinal stromal tumors in Iceland, 1990-2003: the Icelandic GIST study, a population-based incidence and pathologic risk stratification study. Int J Cancer. 2005;117(2):289-293.

3. Nilsson B, Bümming P, Meis-Kindblom JM, et al. Gastrointestinal stromal tumors: the incidence, prevalence, clinical course, and prognostication in the preimatinib mesylate era - a population-based study in western Sweden. Cancer. 2005;103(4):821-829.

4. Fletcher CD, Berman JJ, Corless C, et al. Diagnosis of gastrointestinal stromal tumors: a consensus approach. Hum Pathol. 2002;33(5): 459-465.

5. Joensuu H. Gastrointestinal stromal tumor (GIST). Ann Oncol. 2006; 17(S10):x280-x286.

6. Chen WG, Shan GD, Zhang H, et al. Double-balloon enteroscopy in small bowel tumors: a Chinese single-center study. World J Gastroenterol. 2013;19(23):3665-3671.

7. Tangkittikasem N, Boonyaarunnate T, Aswakul P, Kachintorn U, Prachayakul V. Clinical, radiologic, and endoscopic manifestations of small bowel malignancies: a first report from Thailand. Asian Pac $J$ Cancer Prev. 2015;16(18):8613-8618.
8. Werewka-Maczuga A, Osiński T, Chrzan R, Buczek M, Urbanik A. Characteristics of computed tomography imaging of gastrointestinal stromal tumor (GIST) and related diagnostic problems. Pol J Radiol. 2011;76(3):38-48.

9. Kopáčová M, Rejchrt S, Bureš J, Tachecí I. Small intestinal tumours. Gastroenterol Res Pract. 2013;2013:702536.

10. Xing GS, Wang S, Sun YM, Yuan Z, Zhao XM, Zhou CW. Small bowel stromal tumors: different clinicopathologic and computed tomography features in various anatomic sites. PLoS One. 2015;10(12):e0144277.

11. Joensuu $H$. Risk stratification of patients diagnosed with gastrointestinal stromal tumor. Hum Pathol. 2008;39(10):1411-1419.

12. Wu TJ, Lee LY, Yeh CN, et al. Surgical treatment and prognostic analysis for gastrointestinal stromal tumors (GISTs) of the small intestine: before the era of imatinib mesylate. BMC Gastroenterol. 2006;6:29.

13. Baheti AD, Shinagare AB, O'Neill AC, et al. MDCT and clinicopathological features of small bowel gastrointestinal stromal tumours in 102 patients: a single institute experience. Br J Radiol. 2015; 88(1053):20150085.

14. Sorour MA, Kassem MI, Ghazal A-H, El-Riwini MT, Abu Nasr A. Gastrointestinal stromal tumors (GIST) related emergencies. Int J Surg. 2014;12(4):269-280.

15. Kothari MS, Kosmoliaptsis V, Meyrick-Thomas J. Small bowel gastrointestinal stromal tumors can physiologically alter gut motility before causing mechanical obstruction. Int Semin Surg Oncol. 2005;2:24.

16. Masselli G, Casciani E, Polettini E, Laghi F, Gualdi G. Magnetic resonance imaging of small bowel neoplasms. Cancer Imaging. 2013;13(1): 92-99.

17. Lupescu IG, Grasu M, Boros M, et al. Gastrointestinal stromal tumors: retrospective analysis of the computer-tomographic aspects. $J$ Gastrointestin Liver Dis. 2007;16(2):147-151.

18. Nakatani M, Fujiwara Y, Nagami Y, et al. The usefulness of doubleballoon enteroscopy in gastrointestinal stromal tumors of the small bowel with obscure gastrointestinal bleeding. Intern Med. 2012; 51(19):2675-2682.

19. Martín-Lagos-Maldonado A, Barrientos-Delgado A, MartínezTirado MP, Casado-Caballero FJ. Computed tomography angiography (CTA) in the diagnosis of an infrequent cause of gastrointestinal bleeding. Rev Esp Enferm Dig. 2013;105(2):114-115.

20. Tseng CM, Lin IC, Chang CY, et al. Role of computed tomography angiography on the management of overt obscure gastrointestinal bleeding. PLoS One. 2017;12(3):e0172754.

21. Hersh MR, Choi J, Garrett C, Clark R. Imaging gastrointestinal stromal tumors. Cancer Control. 2005;12(2):111-115.

22. Moawad FJ, Larock TR, Biondi MC, Cash BD, Kurland JE. A case of obscure gastrointestinal bleeding secondary to a small bowel gastrointestinal stromal tumor detected by magnetic resonance enterography. Medscape J Med. 2008;10(11):263.

23. Graça BM, Freire PA, Brito JB, Ilharco JM, Carvalheiro VM, CaseiroAlves F. Gastroenterologic and radiologic approach to obscure gastrointestinal bleeding: how, why, and when? Radiographics. 2010;30(1): $235-252$.

24. He Q, Bai Y, Zhi FC, et al. Double-balloon enteroscopy for mesenchymal tumors of small bowel: nine years' experience. World $J$ Gastroenterol. 2013;19(11):1820-1826.

25. Chen WG, Shan GD, Zhang H, et al. Double-balloon enteroscopy in small bowel tumors: a Chinese single-center study. World J Gastroenterol. 2013;19(23):3665-3671.

26. Brearley S, Hawker PC, Dorricott NJ, et al. The importance of laparotomy in the diagnosis and management of intestinal bleeding of obscure origin. Ann R Coll Surg Engl. 1986;68(5):245-248.

27. Kang YK, Kang HJ, Kim KM, et al. Clinical practice guideline for accurate diagnosis and effective treatment of gastrointestinal stromal tumor in Korea. Cancer Res Treat. 2012;44(2):85-96.

28. Şahin S, Ekinci Ö, Seçkin S, Dursun A. The diagnostic and prognostic utility of DOG1 expression on gastrointestinal stromal tumors. Turk Patoloji Derg. 2017;33(1):1-8. 
29. Koo DH, Ryu MH, Kim KM, et al. Asian Consensus Guidelines for the diagnosis and management of gastrointestinal stromal tumor. Cancer Res Treat. 2016;48(4):1155-1166.

30. Ihn K, Hyung WJ, Kim HI, et al. Treatment results of small intestinal gastrointestinal stromal tumors less than $10 \mathrm{~cm}$ in diameter: a comparison between laparoscopy and open surgery. J Gastric Cancer. 2012;12(4): 243-248.

31. Shen C, Chen H, Yin Y, et al. Duodenal gastrointestinal stromal tumors: clinicopathological characteristics, surgery, and long-term outcome. BMC Surg. 2015;15:98.

32. ESMO/European Sarcoma Network Working Group. Gastrointestinal stromal tumours: ESMO Clinical Practice Guidelines for diagnosis, treatment and follow-up. Ann Oncol. 2014;25 (Suppl 3):iii21-iii26.

33. Nishida T, Blay JY, Hirota S, Kitagawa Y, Kang YK. The standard diagnosis, treatment, and follow-up of gastrointestinal stromal tumors based on guidelines. Gastric Cancer. 2016;19(1):3-14.

34. Dematteo RP, Lewis JJ, Leung D, Mudan SS, Woodruff JM, Brennan MF. Two hundred gastrointestinal stromal tumors: recurrence patterns and prognostic factors for survival. Ann Surg. 2000;231(1):51-58.

35. Rutkowski P, Nowecki ZI, Michej W, et al. Risk criteria and prognostic factors for predicting recurrences after resection of primary gastrointestinal stromal tumor. Ann Surg Oncol. 2007;14(7):2018-2027.

36. Dematteo RP, Ballman KV, Antonescu CR, et al. Adjuvant imatinib mesylate after resection of localised, primary gastrointestinal stromal tumour: a randomised, double-blind, placebo-controlled trial. Lancet. 2009;373(9669):1097-1104.

37. Corless CL, Ballman KV, Antonescu CR, et al. Pathologic and molecular features correlate with long-term outcome after adjuvant therapy of resected primary GI stromal tumor: the ACOSOG Z9001 trial. J Clin Oncol. 2014;32(15):1563-1570.
38. Joensuu H, Eriksson M, Sundby Hall K, et al. One vs three years of adjuvant imatinib for operable gastrointestinal stromal tumor: a randomized trial. JAMA. 2012;307(12):1265-1272.

39. Joensuu H, Eriksson M, Sundby Hall K, et al. Adjuvant imatinib for high-risk GI stromal tumor: analysis of a randomized trial. J Clin Oncol. 2016;34(3):244-250.

40. Poveda A, García del Muro X, López-Guerrero JA, et al. GEIS guidelines for gastrointestinal sarcomas (GIST). Cancer Treat Rev. 2017; 55:107-119.

41. National Comprehensive Cancer Network. NCCN clinical practice guidelines in oncology: soft tissue sarcoma. Version 1.2018. Available from: https://www.nccn.org/store/login/login.aspx?ReturnURL=https:// www.nccn.org/professionals/physician_gls/pdf/sarcoma.pdf. Accessed January 7, 2018.

42. Fu Y, Hao H, Guo L, Yang G, Zhang X. Retrospective analysis of 85 cases of intermediate-risk gastrointestinal stromal tumor. Oncotarget. 2017; 8(6):10136-10144.

43. Lin JX, Chen QF, Zheng $\mathrm{CH}$, et al. Is 3-years duration of adjuvant imatinib mesylate treatment sufficient for patients with high-risk gastrointestinal stromal tumor? A study based on long-term follow-up. J Cancer Res Clin Oncol. 2017;143(4):727-734.

44. Zhao R, Wang Y, Huang Y, et al. Adjuvant imatinib for patients with high-risk gastrointestinal stromal tumors: a retrospective cohort study. Sci Rep. 2017;7(1):16834

45. The National Bureau of Statistics of China [homepage on the Internet] Available from: http://www.stats.gov.cn/english/. Accessed May 6, 2018 .
Therapeutics and Clinical Risk Management

\section{Publish your work in this journal}

Therapeutics and Clinical Risk Management is an international, peerreviewed journal of clinical therapeutics and risk management, focusing on concise rapid reporting of clinical studies in all therapeutic areas, outcomes, safety, and programs for the effective, safe, and sustained use of medicines. This journal is indexed on PubMed Central, CAS,

\section{Dovepress}

EMBase, Scopus and the Elsevier Bibliographic databases. The manuscript management system is completely online and includes a very quick and fair peer-review system, which is all easy to use. Visit http://www.dovepress.com/testimonials.php to read real quotes from published authors.

Submit your manuscript here: http://www.dovepress.com/therapeutics-and-clinical-risk-management-journal 